\title{
Alimentación y cultura en España: una aproximación desde la antropología social
}

| ${ }^{1}$ Mabel Gracia Arnaiz |

Resumo: El hecho alimentario es, esencialmente, multidimensional: transita entre el espacio ecológico, biológico, psicológico, socioeconómico o político. Como ha sucedido con otros fenómenos de naturaleza compleja, hoy nadie discute que la alimentación pueda, o mejor dicho deba, ser estudiada atendiendo a una perspectiva interdisclipinar. En España, son numerosos los trabajos que hechos desde las ciencias experimentales, sociales o humanas dan cuenta, recurriendo a un conocimiento hiperespecializado, de las dimensiones nutricionales, psicológicas o económicas de las preferencias y aversiones alimentarias, del consumo de alimentos o de la obesidad, por poner algunos ejemplos. Se trata, sin embargo, de visiones normalmente unilineales sobre el mismo fenómeno, arropadas por los marcos epistémicos de cada ciencia. La colaboración científica apenas repliega las disciplinas más afines. Escatimar las intersecciones científicas no parece, como trataré de mostrar en este artículo, ni oportuno ni útil. Menos cuando se pretende dar cuenta y resolver problemas que, afectando a los grupos sociales, se desenvuelven en contextos plurales y cambiantes.

> Palavras-chave: alimentación, cultura, antropología social, interdisciplinaridad, España.

1 Profesora de Antropología

Social, Universidad Rovira i Virgili,

España. Correo electrónico:

mabel.gracia@urv.cat

Recibido: 01/02/2010. Aprobado: 10/05/2010. 


\section{Introducción}

Este artículo tiene como fin presentar los estudios de Alimentación y Cultura en España. Sin embargo, son tantos los trabajos realizados y tan diversas las disciplinas implicadas que convierten esta tarea, de no establecerse ciertos límites, en un objetivo excesivamente amplio. A los límites impuestos por estas páginas, yo añado mis inquietudes y competencias, circunscribiendo el objetivo principal del artículo a presentar, como ya hiciera en trabajos anteriores ${ }^{1}$, el campo de estudio de la antropología de la alimentación en España, su emergencia, sus oportunidades y sus límites. Esbozaré, en este intento, algunos puentes han de permitir llevar lo social-como modo particular de aprehender el comportamiento alimentario y de razonarlo - a otras ciencias, a veces excesivamente enfrascadas en los determinismos de la naturaleza y la biología.

La centralidad que la alimentación ha adquirido en la subsistencia humana a lo largo de la historia, y el conjunto de las actividades económicas, tecnológicas, sanitarias, sociales o políticas que la articulan, han hecho de ella un objeto atractivo para la ciencia. En general, la literatura científica muestra el hecho alimentario como un objeto poliédrico de múltiples caras, limitadas entre sí. Cada cara responde, habitualmente, a una visión o interés particular sobre la comida o el comer, sobre sus funciones y efectos. Como ha sucedido con otros fenómenos de naturaleza compleja, hoy nadie discute que lo alimentario pueda, o mejor dicho deba, ser estudiado atendiendo a una perspectiva multidisclipinar. Así, son numerosos los trabajos que hechos desde las ciencias experimentales, sociales o humanas dan cuenta, recurriendo a un conocimiento especializado, de las dimensiones nutricionales, psicológicas o económicas de las preferencias y aversiones alimentarias, del consumo de alimentos o de la obesidad, por poner algunos ejemplos.

Se trata, sin embargo, de visiones normalmente unilineales sobre el mismo fenómeno, arropadas por los marcos epistémicos de cada ciencia. La colaboración científica apenas repliega las disciplinas más afines. Cuando la biomedicina se interesa por el objeto de la alimentación, ésta queda reducida a su capacidad nutricional, concibiéndola únicamente como un agente causal de salud o enfermedad. Cuando, por su parte, la economía le dedica su atención, ésta se circunscribe a evaluar la disponibilidad o el gasto alimentario en un país o la capacidad de generar negocio de un sector. Y así sucesivamente. 
Escatimar las intersecciones científicas no parece, en ningún caso, oportuno. Y menos cuando se pretende dar cuenta y resolver problemas que, afectando a los grupos sociales, se desenvuelven en contextos plurales y cambiantes. Los hechos alimentarios son, esencialmente, transdiciplinares: transitan entre el espacio ecológico, biológico, psicológico, socioeconómico o político. La antropología, como otras ciencias cuyo objeto de estudio camina por espacios intersticiales, ha buscado con mayor o menor éxito -o esfuerzo- favorecer encuentros interdisciplinares, especialmente, aunque no sólo, cuando sus investigaciones han tenido una finalidad aplicada. En favor de ello, ha jugado el haberse interrogado desde sus orígenes acerca del porqué de los elementos comunes y divergentes presentes en las múltiples caras del poliedro alimentario. Aún así, y junto a las relevantes aportaciones de estudiosos como Khare (1980), Messer (1984), Fischler y De Garine (1988) o Hubert (1995), hay que seguir elaborando nuevas propuestas complementarias.

En la actualidad, la (auto)reflexión y, sobre todo, la urgencia ante problemas de subsistencia graves están predisponiendo el diálogo entre las diferentes disciplinas que se ocupan lo alimentario. Se puede observar en los lugares donde han emergido debates teórico-metodológicos intensos, como EEUU, Francia o Gran Bretaña; y en países donde la diversidad socioeconómica y étnica, la situación de hambre y pobreza extrema de ciertas poblaciones y la necesidad de dar respuestas a necesidades concretas han favorecido, con mayor frecuencia, la discusión sobre los límites del propio conocimiento científico y la búsqueda de la colaboración entre estudiosos procedentes de distintas disciplinas. En algunos lugares, como podrán observar los lectores en los dos artículos sobre Brasil y México que acompañan este texto, el estudio de las relaciones existentes entre la Alimentación, Salud y Cultura se ha producido, aunque no sólo, desde el campo de las ciencias biomédicas y, en particular, de la nutrición. En este sentido, son numerosos los investigadores que han optado ampliar, para mejorar sus propuestas de intervención, su formación en ciencias sociales, sobrepasando los límites impuestos por una epistemología obsesionada, a menudo, en medir y cuantificar las ingestas alimentarias y, menos, en comprender la compleja naturaleza de los comportamientos alimentarios. En España, como veremos a continuación, los esfuerzos por aproximar posiciones han sido, hasta la fecha, mucho más limitados y, en cualquier caso, insuficientes. 


\section{Alimentación y cultura:}

un campo de estudio para la antropología social

\section{Del campo y del objeto}

Los estudios de Alimentación y Cultura realizados en España no pueden desligarse de la evolución - y del auge - que este campo de estudio ha tenido en el ámbito internacional (Gracia 1996b). Cuatro son las principales razones por las que, a lo largo de las tres últimas décadas, la antropología social ha ido tomando la alimentación humana objeto de estudio específico. El primer motivo tiene que ver con la constatación de que el estudio de las prácticas alimentarias, a través de sus aspectos materiales, sociales y proyección simbólica, constituye un medio para analizar otras cosas. La supervivencia de un grupo depende en buena parte de la satisfacción de sus necesidades alimentarias, de ahí que sea normal que la búsqueda de comida constituya una de los aspectos más diversos y comunes de cualquier cultura. En este sentido, la producción, distribución y consumo de alimentos, así como el control de todos estos procesos, o las relaciones entre las sociedades y su entorno ha estado en la base de numerosos estudios de antropología económica y ecológica; la selección cuidadosa de alimentos, la ofrenda sagrada de manjares, las interdicciones o las preferencias que recaen sobre ciertas comidas y personas han llenado capítulos de trabajos sobre religión y sistemas de creencias; la comida como medio para el establecimiento derechos y obligaciones entre familiares, parientes o amigos o como forma de manifestar agradecimiento, compromiso, deuda o cariño han sido temas tratados por la antropología del parentesco; del mismo modo, el vocabulario alimentario, así como los campos semánticos o las taxonomías culinarias han sido atendidos desde la antropología cognitiva en su interés por demostrar ciertas relaciones entre lenguaje, pensamiento y realidad y en su interés por destacar el valor de las categorías "emic" o el punto de vista de los actores sociales; la ritualización y las fiestas, la comensalidad, los recetarios, los calendarios de actividades y comidas, los productos de la tierra, la restitución o la invención de nuevos formas de abastecer los mercados ha llamado, a su vez, la atención de los estudiosos del patrimonio cultural y de los estudios regionales; del mismo modo, los alimentos como fuente de salud y enfermedad, de males y remedios, de temores y obsesiones han tenido un lugar también en los trabajos de antropología de la medicina. 
En general, pues, el estudio de los roles desempeñados por los alimentos y la comida en el establecimiento y la manifestación de relaciones sociales ha sido más o menos constante en la mayor parte de las diferentes áreas de estudio de la disciplina. De este modo, la antropología social ha ido demostrando de forma más o menos precisa que la alimentación constituye una especie de ventana con vistas a través de la cual observar, conocer y tratar de comprender la articulación de un entramado cultural más amplio. El hecho alimentario es, en el sentido dado por Mauss (1950), un hecho social total, entendiendo que todas las áreas de la cultura y tipos de instituciones (económicas, legales, políticas, religiosas, etcétera) encuentran en él expresión simultánea y le influyen de algún modo. Consecuentemente, el análisis del hecho alimentario puede revelarnos, a su vez, la naturaleza y la estructura de un orden social dado. El sistema alimentario depende de y afecta al resto de sistemas -económico, político, familiar, cultural- que están articulando cada realidad social, de forma que es impensable hacer un análisis de la cultura alimentaria sin vincularlo, necesariamente, con el reparto de poder y autoridad dentro de la esfera económica y política y, en consecuencia, también con el sistema de estratificación social y la división sexual y social del trabajo.

En segundo lugar, la atención de la antropología social hacia la alimentación humana ha tenido que ver con el interés que otras disciplinas han demostrado por este tema y con la circunstancia de que estas áreas de conocimiento en gran parte de base biomédica pero también social hayan convertido la producción, la distribución y el consumo de alimentos en objeto de interés científico por sus implicaciones en la salud, en los procesos afectivos y cognitivos o en el desarrollo económico y social de las poblaciones. Desde una perspectiva médica, el interés por la alimentación no es una cuestión reciente. La ciencia nutricional, por ejemplo, surge a mediados del siglo XIX, estimulada por los problemas prácticos que afectaban a la salud de la población en relación con la calidad de los alimentos producidos - a menudo adulterados -, por su almacenamiento y las dificultades en el transporte a larga distancia o por los problemas de escasez y enfermedad reinantes entre los trabajadores europeos hacinados en los suburbios de las ciudades (MENNELL et al. 1992). Los problemas de salud hoy se han traslado en estos contextos desde aquellos relativos a la desnutrición, tales como el raquitismo, a los vinculados con la sobrealimentación, aunque siguen subsistiendo con características parecidas en los países pobres. La importancia de la nutrición 
ha ido creciendo al ritmo rápido que lo ha hecho el multifacético poder médico y hoy los nutricionistas están inmersos en todas las organizaciones estatales e internacionales prescribiendo recomendaciones dietéticas y patrones de conducta en cualquier parte del mundo. De esta posición eminente ha resultado en las dos últimas décadas un incremento notable de la recopilación de datos a nivel nacional sobre los modelos de consumo alimentario y una mayor investigación de base epidemiológica.

Desde una perspectiva social, el interés más reciente procede de diferentes disciplinas. La escuela francesa de historia de los Annales, especialmente en los años sesenta y setenta y a partir del trabajo de Braudel no ha dejado de interesarse por todos los aspectos de la vida material, situando en un lugar destacado la alimentación. Del mismo modo, desde la perspectiva de economía y ecología política los sistemas alimentarios y los conceptos asociados de redes, regímenes y estructuras alimentarias o agroecosistemas han sido objeto de una fructífera atención empírica y teórica en los veinte últimos años. Por su parte, el giro culturalista producido en las ciencias sociales en la década de los años noventa a partir de la influencia creciente de los enfoques postestructuralistas o de tradiciones como los estudios culturales ha hecho que en algunas disciplinas, como la sociología o la psicología, se empiecen a dar más importancia a los aspectos cotidianos de la cultura -la cultura popular- y a utilizar con más frecuencia las metodologías cualitativas de corte etnográfico.

En el contexto actual de producción de mercancías, los alimentos han ido adquiriendo una centralidad enorme, tanto por el valor de uso y/o de cambio que a lo largo de la historia y de las culturas ha tenido para el sustento y el intercambio social, como por el rol económico central que ha ido adquiriendo en la sociedad contemporánea. Las disparidades mundiales referentes al abastecimiento y la accesibilidad de los alimentos amenazan con no resolverse en un futuro próximo y hoy se habla de la segmentación del planeta (norte/sur, ricos/pobres, primer/ tercer mundo) en términos alimentarios: aquellos que acceden más o menos fácilmente a la comida, y en cuyas rentas la partida destinada a la alimentación es cada vez menos significativa, y aquellos que no saben qué comerán mañana si es que hoy han comido algo, o que lo hacen gracias a la "bondad" y a las ayudas de los primeros; aquellos que enferman por comer en exceso y aquellos que también enferman o se mueren por no hacerlo. De este modo, el interés por el conjunto 
de los hábitos alimentarios se ha ido incorporado en las políticas de salud pública y en los esfuerzos para reducir ciertas enfermedades que, como el caso de las cardiovasculares en los países industrializados o los estados carenciales en los países en desarrollo, son la principal causa de muerte prematura.

El alimento se ha convertido poco a poco en una mercancía y la gran distribución ha dado paso al comedor-consumidor. Los alimentos son hoy más que nunca un negocio próspero para la globalización económica: para las afluentes e influyentes industrias agropecuarias, para numerosos productores y distribuidores, para algunos estados. Nadie duda tampoco que el interés por la comida de otros lugares constituye un gran negocio internacional. Así en España, la actividad agroalimentaria aporta el 6,8 por ciento de la riqueza generada anualmente, proporciona ocupación a un millón y medio de personas (10,6 por ciento de la masa laboral del país) y origina el 14 por ciento de los intercambios totales de mercancías con el exterior (importaciones y exportaciones) (MAPYA, 2001, p. 47). Por su parte, el gasto total en alimentación y bebidas ascendió en 2006 a 81.916 millones de euros (MERCASA, 2007).

Ver en los lineales del supermercado frutas tropicales exóticas, a veces producidos en nuestro propio país, o productos menos exóticos pero pescados o fabricados en otros lugares del mundo es ya algo habitual. Los conflictos creados entre productores y distribuidores para hacerse con los emplazamientos para construir grandes superficies o por aplicar la ingeniería genética a ciertos alimentos para hacerlos más atractivos y/o resistentes a los plaguicidas constituyen algunas de las manifestaciones más evidentes de la punta del iceberg de la industria alimentaria. Por su parte, el alcance social de las sucesivas alarmas o crisis alimentarias hubieran sido impensables unas décadas atrás. Paradójicamente, los nuevos alimentos industriales son también una esperanza: se espera el milagro tecnológico y económico que, sin demasiado malos menores, produzca alimentos superdotados, cuya calidad extrema y composición nutricional resuelva no sólo el problema mundial que mejor refleja la desigualdad social y económica entre países y entre personas, el hambre, sino las dudas existenciales que millones de individuos no hambrientos tienen acerca de lo que comen. La nueva gama de alimentos funcionales nacen provistos de atributos saludables. La ciencia, y también la economía, nos dicen que estamos más cerca de hacer realidad la osmosis alimento/medicamento y que, de alguna manera, la comida acabará permitiendo no ya nuestro deseo de permanecer inmortales, de 
momento poco probable, sino de controlar lo humanamente controlable: algunos aspectos de nuestra salud y bienestar, algunos aspectos de nuestro cuerpo, algunos aspectos de nuestro espíritu.

El vínculo histórico entre alimentación y salud, unido a la aparición cada vez más recurrente de los asuntos alimentarios en las agendas políticas de los años noventa, ha hecho que todos nos preocupemos cada vez más por nuestra dieta y de alguna manera por nuestros comportamientos alimentarios. El tercer motivo que ha suscitado la atención de la antropología social por la alimentación está relacionado, pues, con este aumento del interés general mostrado por la población y con el hecho de que la alimentación se reconozca como un elemento articulador de un buen número de prácticas y representaciones sociales y también de problemas. Esta centralidad del hecho alimentario ha vitalizado, sin duda, la expansión paralela de la investigación académica y, en particular, los estudios antropológicos y reconocemos que estuvo en buena parte en el origen de nuestro interés por este tema. El comer y el no-comer expresan numerosos significados. En los medios de comunicación podemos encontrar con un cierta regularidad y frecuencia, sobre todo si comparamos con los contenidos periodísticos de cincuenta años atrás, desde programas de cocinas especializadas o remedios caseros para ciertos males, documentales de viajeros narrando las peculiaridades de cocinas exóticas, a debates sobre la salud y la inocuidad o no de los alimentos y sus repercusiones en nuestro cuerpo o en el medio ambiente, mientras que en los rankings editoriales aparecen libros de regímenes de adelgazamiento como best-sellers. De forma paralela, este auge se ha visto impulsado por la proliferación y aceptación de las denominadas cocinas étnicas o temáticas, las cuales han llegado desde lugares remotos hasta ciudades de cualquier parte del mundo a través de platos más o menos estandarizados y/o inventados, que han contribuido así a nutrir la ya abundante oferta restauradora y el imaginario exótico de las personas más neófilas.

Finalmente, una cuarta razón por la que la antropología social ha focalizado su interés creciente en la alimentación y la comida tiene que ver con un cambio habido en el centro de gravedad de la antropología misma, que nos lleva desde los procesos de producción, distribución y consumo alimentario y sus consecuencias en la dinámica social, económica y política de las culturas tradicionales, al análisis de la organización del consumo y de los fundamentos materiales y simbólicos de este proceso en cualquier sociedad. En las dos últimas décadas, 
cada vez más antropólogos han dejado de observar los estudios sobre consumo con recelo porque en ellos se abordaban los efectos de la introducción de los bienes de consumo industriales en las sociedades tradicionales, lo que podía llevar implícito la aceptación de la pérdida o disolución de las culturas no modernas y, consecuentemente, el fin del propio objeto de estudio de la antropología. Contrariamente, durante este periodo se han ido incrementando los trabajos que atendiendo precisamente a la entrada masiva de los productos derivados de la modernización en estas y otras sociedades están dando cuenta de las complejas relaciones surgidas entre los procesos de alcance global y las respuestas e interpretaciones locales. En este contexto, los alimentos y en un sentido más amplio los "productos alimentarios" en tanto que bienes de consumo masivo han dado otro empuje a la agenda antropológica. Lo mismo puede decirse del valor adquirido por los temas basados en las experiencias de las mujeres a partir de los estudios y teorías producidas sobre todo desde la perspectiva de género. El aprovisionamiento, la preparación y el servicio de los alimentos han sido y son actividades diarias principalmente femeninas vinculadas en buena parte al trabajo doméstico. El hecho de que la esfera doméstica y la vida cotidiana hayan adquirido una mayor relevancia en los estudios antropológicos ha beneficiado también la atención por estos temas previamente desestimados por el escaso interés en casar el pensamiento científico con elementos en apariencia tan intrascendentes como un plato de legumbres o una técnica para hacer conservas.

\section{Antropología de la alimentación}

Si aceptamos que la cultura alimentaria ${ }^{2}$ tanto crea como refleja la especificidad humana y su diversidad, entenderemos fácilmente que se haya convertido en objeto de estudio para la antropología, aunque la preocupación científica por la alimentación sea un fenómeno relativamente reciente desde el punto de vista antropológico. Más aún si nos remitimos a España, donde esta ciencia se ha desarrollado con posterioridad respecto a otras tradiciones europeas y americanas (GRACIA, 2002). En realidad, lo que es más relativamente reciente no es tanto el interés por el análisis social de la comida, como los esfuerzos de carácter teórico y metodológico que se han llevado a cabo durante las tres últimas décadas para definir lo que ha venido denominándose antropología de la alimentación - anthropology of food: la disciplina dedicada al estudio de las prácticas y 
representaciones alimentarias de los grupos humanos desde una perspectiva comparativa y holística. La antropología de la alimentación pone su atención sobre todo en los factores ecológicos, socioculturales, económicos o filosóficos que influyen en la elección de los alimentos, teniendo en cuenta, sin embargo, que existen otros de carácter biológico y psicológico que interaccionan entre sí.

Los antropólogos desde hace ya más de un siglo han manifestado su interés por el estudio sociocultural de la alimentación al registrar con mayor o menor énfasis la extraordinaria carga comunicativa que todos los grupos sociales generan en torno a las comidas. Ha sido, no obstante, una dedicación oscilante y dispersa, según el momento, el lugar y las corrientes teóricas que se han ido sucediendo en la antropología, tal como indican los investigadores sociales que se han dedicado a recoger, ordenar y discutir de forma exhaustiva el conjunto de trabajos realizados a largo del siglo XX sobre alimentación y cultura. ${ }^{3}$ Dicho conjunto de trabajos nos permite establecer un recorrido teórico y metodológico que va desde los primeros enfoques evolucionistas, que pusieron la atención en los aspectos rituales y sobrenaturales del consumo de los alimentos y, especialmente, en todas las costumbres "extrañas" capaces de explicar la evolución de las instituciones sociales, hasta los recientes enfoques de la economía política o las corrientes postestructuralistas, centrados en analizar los efectos de la globalización en los sistemas alimentarios locales o los aspectos más problemáticos de la alimentación y nutrición: el hambre, las innovaciones tecnológicas, la obesidad o los transtornos del comportamiento alimentario, la seguridad alimentaria o la desestructuración de las maneras de comer.

Una buena parte de estos trabajos sugiere también la necesidad metodológica de aproximarse al estudio del comportamiento alimentario a través de la etnografía como una vía eficaz para abordar de forma comparativa y holista la compleja naturaleza de dicho comportamiento y de sus aspectos más relevantes y significativos, dado que prioriza todos aquellos instrumentos analíticos que favorecen la observación, la descripción y el análisis de la vida cotidiana de los miembros de una sociedad dada: observación participante, apuntes biográficos, descripción, entrevistas, grupos de discusión, diario de campo, apuntes dietéticos, calendario anual o registro audiovisual, etcétera. El hecho alimentario es complejo porque aglutina aspectos variados, unas veces relacionados con la ética y la religión, otros con la diferenciación social, la discriminación y la desigualdad, otros con el 
grado de satisfacción individual o social, el sentimiento de identidad colectiva o, en fin, con la sociabilidad, la adaptación o la supervivencia.

Estos múltiples aspectos, participando de la esfera tanto íntima como pública de las personas, son a menudo transformados por la oralidad y por los discursos que construyen los informantes, de tal manera que se hace necesario recoger la información mediante recursos analíticos que permitan la contrastación. A la etnografía le interesa, sin embargo, ver cómo se construye dicha oralidad y no sólo porque a través de ella se expresa el punto de vista interno de los actores sociales o emic, extraordinariamente útil en la tarea de comprender lo que es verdaderamente significativo para el grupo estudiado, sino porque su relato da cuenta de cómo los actores sociales organizan el conocimiento y sus experiencias alimentarias a través del lenguaje, cómo las perciben y cómo las comunican. Así pues, a la antropología de alimentación le interesa abordar el decalage que pueda existir entre la visión que las personas proporcionan de sus prácticas alimentarias, y lo que realmente hacen: una diferencia no siempre fácil de reconocer y valorar cuando el contacto que se produce entre interlocutor y observador se reduce a escasos minutos de diálogo o a supervisar el inventario de registros alimentarios. En este sentido, cualquier investigación que trate de evaluar, medir y cuantificar el consumo, debe preguntarse previamente si existe algún tipo de especificidad en relación con la alimentación que potencie el desajuste entre el discurso mantenido por la gente, es decir, entre el relato/las respuestas de la persona entrevistada y sus prácticas reales y, de ser así, disponer de los instrumentos útiles para abordarla e interpretarla.

La mayoría de estos trabajos coinciden también en criticar los estudios socioculturales sobre alimentación que en primera instancia y, con frecuencia en única instancia, emplean herramientas de carácter cuantitativo para la recogida y análisis de la información, ya que se ven muy limitados a la hora de dar respuesta al sinfín de porqués que normalmente se generan en torno al comportamiento alimentario. Numerosos cuestionarios cerrados que no se han hecho sobre la base de una aproximación cualitativa previa incluyen un riesgo metodológico importante consistente en establecer generalizaciones a partir de las respuestas a cuestiones que sólo responden al discurso generado por el diseño de las mismas preguntas. Este tipo de encuestas suele ser insuficiente, sobre todo si éstas no se contrastan ni se construyen teniendo en cuenta la dimensión cualitativa. Las variaciones entre el comportamiento alimentario real, la visión que dan y que construyen 
los entrevistados, están llenas de significación acerca de la lógica alimentaria y se escapan fácilmente a los valores "medios" resultantes de los análisis exclusivamente cuantitativos. Por esta razón, la antropología contemporánea subraya la conveniencia de que cualquier estudio sobre comportamiento alimentario vaya precedido y se complemente con técnicas que permitan el análisis directo de las prácticas y de la asignación de significación. Las investigaciones estadísticas pueden sernos de gran utilidad siempre y cuando la sistematización del proceso para la obtención de datos no deje al margen aspectos importantes para la generalización. En este sentido, a esta subdisciplina le interesa menos concluir acerca de qué come exactamente un grupo social determinado, que acerca de por qué come uno u otro alimento, para qué, con quien, cómo o cuando.

\section{Antropologia de la alimentación en España \\ El interés por los estudios socioculturales sobre alimentación}

Si el estudio de la alimentación no es nuevo para la antropología en general, lo es algo más para la antropología española, ya que discurre en paralelo a la mayor implantación de esta disciplina en el ámbito académico y al auge que experimenta, especialmente a partir de la década de los ochenta, en países como Francia, Estados Unidos o Gran Bretaña. En tanto que se trata de un interés relativamente nuevo no es fácil evaluar los resultados que, poco a poco, se han ido generando alrededor de las diferentes líneas de investigación abiertas. No obstante, voy a tratar de presentarlos, aunque sea de forma sucinta.

Son numerosos los estudios realizados en España que, vinculándose a ámbitos de la gastronomía y la comensalidad festiva, el folklore y las tradiciones populares, la historia oral o el patrimonio, han abordado el estudio de algún aspecto de la cultura alimentaria o han tratado facetas particulares del sistema alimentario a través del análisis de la producción, transformación distribución o consumo de alimentos. ${ }^{4}$ Cabe señalar, sin embargo, que a pesar de su interés, no todos estos trabajos han tomado lo alimentario teniendo en cuenta las discusiones teórico-metodológicas que impregnan el debate socioantropológico (Contreras y Gracia, 2005) ni se han interesado por las generalizaciones e hipótesis contenidas en el embrionario núcleo teórico que a nivel internacional se ha generado en torno a la antropología de la alimentación. En es sentido, y como sucede en otros países, sigue siendo una realidad lo que ya señalaron a principio de los noventa Mennell et al. (1992) y Carrasco 
(1992): una parte significativa de los trabajos son principalmente descriptivos y

se han ido sucediendo, salvo excepciones, sin ninguna validación decisiva de las aproximaciones, interrogantes o respuestas generadas con anterioridad. No obstante, los esfuerzos de antropología española por delimitar y definir el objeto de estudio y la construcción de un núcleo teórico y metodológico más sólido no son tampoco desestimables. A lo largo de la última década, el reconocimiento académico de los estudios de Alimentación y Cultura se ha ido materializando de diversas formas, coincidiendo con el incremento de los problemas -y de los intereses- que suscita en el actual orden económico y sanitario internacional/local lo alimentario y con la dificultad de encontrar respuestas a las cuestiones que ciertas prácticas plantean respecto a la salud, el medio ambiente y la economía. En este contexto, todo parece indicar que nos hallamos ante un ámbito disciplinar con posibilidades de arraigarse en el tejido social, sobre todo a través de las iniciativas adoptadas en educación superior y en investigación básica y aplicada.

De forma específica, las razones que están contribuyendo al desarrollo de la antropología de la alimentación en España durante los últimos veinte años son principalmente tres: la introducción de cursos monográficos en diversas carreras universitarias, la creación de grupos de investigación con soporte institucional y de redes temáticas y el incremento de las publicaciones científicas. En efecto, asignaturas con el título de "Alimentación y Cultura", "Antropología de la alimentación", "Alimentación y Cambio Social", "Antropología Alimentaria", "Alimentación, Nutrición y Cultura", "Alimentación y Creencias", "Historia de la Alimentación" o "Alimentación, Salud y Cultura" que tienen como objetivo principal abordar la vertiente social de la alimentación humana, vienen impartiéndose hoy en diplomaturas, licenciaturas, màsters y terceros ciclos de diferentes áreas de estudio de las universidades españolas, tales como Antropología Social, Historia, Sociología, Ciencia y Tecnología de los Alimentos, Nutrición Humana y Dietética.

Durante el mismo período, han aparecido múltiples grupos de investigación repartidos en el mapa académico español, respondiendo a iniciativas surgidas, sobre todo aunque no sólo, en el ámbito universitario5. De características y composición diversa, la mayoría dedica notables esfuerzos a conseguir recursos para la financiación de sus proyectos a través de ayudas, contratos o convenios establecidos con instituciones públicas y privadas autonómicas, estatales e internacionales. Las investigaciones llevadas a cabo por estos grupos responden a intereses particulares 
(con frecuencia tesis doctorales) o colectivas. Las líneas principales temáticas abordadas han discurrido en torno a los siguientes ejes:

- Enculturación y transmisión saber-hacer alimentario: procesos de socialización, institucionalización del aprendizaje alimentario, cocina doméstica y profesional, adquisición de gustos, preferencias y aversiones alimentarias

- Cocinas regionales, patrimonio etnológico y desarrollo local: tipologías culinarias, recursos ecológicos y socioeconómicos, caracterización de los productos de la tierra, sostenibilidad y desarrollo rural, identidades culturales, patrimonio alimentario y turismo

- Evolución del sistema alimentario y maneras de comer: influencia factores estructurales en cambios alimentarios, flujos e intercambios alimentarios, reformas agrarias, glocalización, políticas alimentarias, , nuevos mercados, mercancías y consumidores, crisis alimentarias, movimientos sociales alternativos (ecologistas, neorrurales, vegetarianos, tierras sin OGMS)

- Alimentación y desigualdad social: producción alimentaria y geopolítica, acceso y disponibilidad de alimentos, pobreza y hambre, soberanía alimentaria y empoderamiento

- Prácticas y representaciones alimentarias: culturas alimentarias, comestible/no comestible, religiones y creencias alimentarias, percepción de las innovaciones tecnológicas, seguridad alimentaria, alimentos funcionales, AGMs; discursos publicitarios

- Movimientos migratorios y alimentación: interculturalidad y mestizajes, procesos de adaptación, cocinas de fusión

- Alimentación, salud y cultura: malnutrición y desigualdad social, políticas alimentarias en salud, medicalización de la alimentación, dimensiones socioculturales de la obesidad, TCA.

Buena parte de los grupos que se han ido consolidando en el panorama español, cuentan con investigadores procedentes de áreas de conocimiento distintas a la antropología. Es el caso de la arqueología, la historia, la sociología, la filología, la restauración, la geografía, la nutrición y la dietética, la bromatología o el arte. Sin embargo, ello no se ha traducido, necesariamente, en la realización de estudios de base interdisciplinar. Habitualmente, estos equipos acostumbran a trabajar de forma independiente, aunque también se han hecho investigaciones intergrupales. 
Entre ellos, en Cataluña destaca el Observatorio de la Alimentación ${ }^{6}$, un grupo de investigación consolidado del Parque Científico de Barcelona (Universidad de Barcelona) El ODELA constituye un equipo interdisciplinario e interuniversitario integrado por investigadores interesados en el análisis y la comprensión de los comportamientos alimentarios desde una perspectiva histórica, social, económica y cultural. Ha centrado sus investigaciones en diferentes áreas de la cultura alimentaria, abordando tanto su realidad presente como su dimensión histórica. Entre sus proyectos principales, destacan las investigaciones sobre los productos de la tierra en el sur de Europa y Cataluña, los factores sociocognitivos que influyen en las elecciones alimentarias según las diferentes edades, formas de aprovisionamiento alimentarios y Tercera Edad, la percepción social de la biotecnología o la caracterización de las maneras de comer contemporáneas. Una iniciativa interesante de la Universidad de Barcelona ha sido incluir el Master Bienal Europeo en Historia y Cultura de la Alimentación en el Programa Oficial de Postgrado de Alimentación y Nutrición, junto a estudios vinculados con Tecnología de los Alimentos y Dietética apostando, así, por la pluridisciplinaridad y el concurso entre las ciencias experimentales y sociales.

También en Cataluña hay que situar el equipo de investigación consolidado Grupo de Investigación en Antropología Social, Filosofía y Trabajo Social (GIAFITS) de la Universidad Rovira i Virgili (Tarragona), el cual está formado, principalmente, por antropólogos, filósofos y trabajadores sociales. Dentro de este grupo, hay investigadores que, vinculados a la Red de Antropología Médica (REDAM), ${ }^{7}$ se han interesado por analizar las relaciones entre género, cultura y alimentación, han estudiado las dimensiones culturales de los trastornos del comportamiento alimentario y la obesidad, así como la influencia de las políticas alimentarias en salud y en el proceso de medicalización de la alimentación. Entre sus aportaciones, destacan también los estudios sobre el análisis de los factores estructurales en las maneras de comer actuales, la percepción del riesgo alimentario o las narrativas sobre cuerpo y comida en Internet.

Por su parte, en Barcelona hay que ubicar a la Societat per a l'Estudi Interdisciplinari de l'Alimentació i els Hàbits Socials (SEIAHS). ${ }^{8}$ Se trata de una sociedad de investigación privada constituida en el año 2005 en la que colaboran investigadores catalanes especializados en el anàlisis de la alimentación y otros fenómenos sociales procedentes de áreas de conocimientos diferentes: dietética 
y nutrición, historia, antropología y psicología. Los estudios realizados tienen una orientación principalmente aplicada. Dentro de los objetivos generales del grupo, destaca su interés por promover el conocimiento de la historia y cultura alimentaria, contribuir a favorecer la adquisión de hábitos alimentarios y estilos de vida saludables y aumentar el conocimiento sobre el consumo alimentario, sus efectos en la salud humana y sobre su vínculo con otras dimensiones de las conductas individuales y sociales.

Por su parte, en Andalucía, los principales grupos de trabajo se encuentran enclavados en Córdoba y Sevilla. En la Universidad de Córdoba está el grupo investigación Cultura Alimentaria Andalucía-América de la Facultad de Filosofía y Letras, el cual forma parte del Plan Andaluz de Investigación. Este equipo nació a raíz de la celebración en 1992 de un congreso internacional de Etnobotánica en homenaje a los pueblos indígenas americanos y desde entonces ha venido organizando diversos simposios internacionales sobre aspectos específicos de la cultura alimentaria. Su línea de trabajo se ha focalizado en el estudio diacrónico de la alimentación en Andalucía, España y Latino-América. Le interesa los sistemas alimentarios, o los alimentos y su contexto social e histórico, desde la baja Edad Media hasta la actualidad. Como en los casos anteriores, el equipo tiene una base interdisciplinar constituida por historiadores, filólogos y antropólogos.

La Universidad de Sevilla, por su parte, acogió hasta el año 2002 el grupo Sistemas Alimentarios e identidad cultural. Durante más de una década, este equipo de investigadores trabajó en diversos ámbitos de estudio, principalmente en el estudio de la evolución de los hábitos alimentarios, los sistemas culinarios y tipologías, el patrimonio agroalimentario andaluz, la alimentación mediterránea, la seguridad alimentaria, la historia de la restauración o la maternidad y la asistencia. A raíz de la jubilación de los investigadores principales, esta línea de trabajo se ha incorporado en el grupo Patrimonio Etnológico, Recursos Socioeconómicos y Simbolismo (PERSES) adscrito al Plan Andaluz de Investigación (PAI) de la Junta de Andalucía, el cual está formado por antropólogos.

En Aragón, se encuentra el Equipo de Investigación Multidisciplinar en Alimentación Humana (EIMAH) de la Universidad de Zaragoza9. Este grupo cuenta con la colaboración de antropólogos, tecnólogos de la aimentación, sociólogos, médicos, geógrafos y cocineros y las líneas de investigación activas giran en torno al estudio de las relaciones entre identidad sociocultural y 
aimentación, globalización/glocalización alimenaria, horticultura urbana y ecología y sostenibilidad.

La existencia de estos equipos de investigadores y, en particular, la diversidad de las líneas de trabajo abiertas, constituyen una muestra de que, en efecto, estamos ante un campo de estudio fructífero, en el que ya se han empezado a dar las primeras colaboraciones interuniversitarias e interinstitucionales, no sólo con la intención de favorecer la investigación en las áreas de influencia de los respectivos equipos, sino de ubicarla en un contexto más amplio. Hasta la fecha, sin embargo, no se ha ideado un mecanismo específico de articulación común de estos grupos. Aún así, la creación de redes y grupos estatales o internacionales constituye una muestra del interés, y de la necesidad, que la mayoría de los miembros de estos equipos manifiestan por conocerse entre sí y por trabajar conjuntamente.

En esta línea, se ha creado la red española asociada a la sección europea de la Internacional Comission of Anthropology of Food (ICAF) ${ }^{10}$ que pertenece, a su vez, a la International Union of Anthropological and Ethnological Sciences (IUAES), entre cuyos objetivos principales figura dar soporte a la investigación básica y aplicada en antropología de la alimentación, así como estimular la formación en este campo y las reuniones interdisciplinares. Una de las actividades más consolidadas de ICAF es la organización de simposios internacionales de carácter anual o bianual que giran sobre temas específicos de la cultura alimentaria. A dicho organismo están suscritos alrededor de veinticinco investigadores españoles, aunque no aglutina a todos los estudiosos que en estos momentos están trabajando sobre este ámbito de estudio ni ejerce la función de coordinar los distintos grupos entre sí. También recientemente, en 2008, ha surgido el Grupo de Sociología de la Alimentación ${ }^{11}$, perteneciente a la Federación Española de Sociología, con el objetivo aglutinar a investigadores de la alimentación en su intersección con la salud, la cultura, el consumo y los sistemas agrarios. El grupo acoge también a antropólogos, agrónomos, médicos y otros investigadores, dejando clara la multidisciplinariedad de las aproximaciones al análisis de los cambios alimentarios. Como medio de contacto principal, dispone, dentro de la Red Iris, de la lista denominada SocAliment, que difunde información sobre los estudios que relacionan los cambios sociales con los cambios alimentarios.

No cabe duda de que la impartición de los nuevos cursos universitarios y la creación de grupos y redes temáticas ha beneficiado la implantación progresiva de la 
antropología de la alimentación en España. Todo hace pensar, pues, que de existir una coordinación eficaz, este ámbito de estudio podría consolidarse en un futuro. Uno de los medios que mejor indican la trayectoria de esta área de estudios y sus primeros resultados son las numerosas publicaciones de base compilatoria, teórica y empírica que, escritas en castellano u otras lenguas oficiales del estado español, han visto la luz durante este período ${ }^{12}$. A pesar de la extraordinaria diversidad temática, estos trabajos participan de tres características comunes: están planteados desde una misma curiosidad disciplinar, analizan parcial o globalmente los factores y las variables que, en el contexto de procesos históricos dinámicos y más amplios, explican las tendencias alimenarias y muestran los aspectos diferenciales de las prácticas materiales y simbólicas de la alimentación, sean éstas de orden religioso, dietético étnico, de clase, de edad o género. Además, aunque a nivel metodológico se han resuelto de maneras diferentes, todos consideran, siguiendo las recomendaciones de I. de Garine (1980) o Menell et al. (1992) ${ }^{13}$, que el análisis etnográfico, sociológico e histórico son una vía necesaria para orientar y contrastar las generalizaciones obtenidas por los estudios exclusivamente de base cuantitativa.

Las publicaciones de base compilatoria, por ejemplo, han recogido según los casos, artículos sobre aspectos históricos, antropológicos, geoeconómicos y literarios de la alimentación principalmente en la Península Ibérica, Sudamérica y Europa. Algunos textos han aparecido en forma de monográficos de revistas - El Folk-Lore Andaluz, 1992 (9); Revista d'Etnologia de Catalunya, 1993 (2), 2000 (20), 2005 (27); Estudis d'Història Agrària, 2000 (13); Abaco: Revista de Cultura y Ciencias Sociales, 2002 (31). En ocasiones, derivado de las necesidades docentes, se trata textos de autores extranjeros de obligada referencia que han sido traducidos al castellano, conviertiéndose en manuales útiles para los estudiantes de los ciclos superiores (CONTRERAS, 1995) o de compilaciones de los primeros estudios sobre Alimentación y Cultura hechos por antropólogos en España (Gracia 2002). Por su parte, están los trabajos que, partiendo de las aportaciones de la literatura internacional, han abordado las relaciones entre Alimentación y Cultura (CRUZ, 1991; GARRIDO, 1995, 2001; GUIDONET, 2007) y han debatido sobre las aportaciones teórico-metodológicas de la antropología y sociología al estudio sociocultural de la alimentación (CARRASCO, 1992; CONTRERAS, 1992; GRACIA, 1996b, 2002; MILLÁN, 1999; GAONA; NAVAS, 2003; CONTRERAS; GRACIA, 2005; DÍAZ; GÓMEZ, 2005). También están 
aquellos otros que incluyen diferentes puntos de vista disciplinares sobre una misma cuestión, como es el caso de la alimentación mediterránea o atlántica (MEDINA, 1996; GONZÁLEZ TURMO; ROMERO DE SOLÍS, 1996; CASTRO, 1998; CONTRERAS; RIERA; MEDINA, 2005; GONZÁLEZ TURMO et al., 2007), o aquellos textos de antropólogos españoles que aparecen en obras de base compilatoria no específicamente de alimentación.

Con frecuencia, y como resultado de la realización de tesis doctorales o investigaciones colectivas han aparecido también libros sobre facetas particulares de la cultura alimentaria en España, destacando aquellos sobre las diferencias entre cocinas populares y de élite (GONZÁLEZ TURMO, 1995), la construcción del saber-hacer alimentario (GRACIA, 1996a), las relaciones entre cambio social y cambio alimentario (GRACIA, 1997), la migración y los cambios de alimentación (KAPLAN; CARRASCO 1999; MEDINA, 2002; CÁCERES; ESPEITX, 2006) o el patrimonio etnológico alimentario (ESPEITX; CÁCERES; MASSANÉS, 2001; CONTRERAS; CÁCERES; ESPEITX, 2003). Dentro de este grupo, hay que ubicar las obras relacionadas con las maneras de comer actuales de la población española, las cuales han intentado caracterizar tanto las jornadas alimentarias como la influencia de los factores contextuales que condicionan la naturaleza compleja de los comportamientos alimentarios (CONTRERAS; GRACIA, 2004; DÍAZ, 2005; MEDINA, 2005; CONTRERAS; GRACIA, 2006). Por último, es preciso señalar que, en contexto de la literatura española, las relaciones sobre alimentación, salud y cultura han recibido una atención especial por parte de los investigadores sociales, siendo un ejemplo los trabajos compilatorios de base interdisciplinar que han ocupado monográficos de revistas o de colecciones de libros (GRACIA; MILLÁN, 2005; DÍAZ; GÓMEZ, 2005; DÍAZ; GÓMEZ, 2008) o los que han abarcado aspectos más específicos. Es el caso, por ejemplo, del estudio de las dimensiones culturales de los trastornos del comportamiento alimentario (ISOLETTA, 2003; GRACIA; COMELLES, 2007; GRACIA, 2007) o de la relación entre alimentación, salud y clases populares (MARTÍN; MORENO, 2005).

\section{Oportunidades, necesidades y límites: ¡un futuro para la antropología de la alimentación en España?}

El hecho de que la antropología de la alimentación sea en España un campo de estudio fructífero no implica la ausencia de problemas de fondo que, de 
momento, parecen limitar más que ayudar a la expansión de esta área. Hay que admitir, de entrada, que el campo de la antropología de la alimentación no juega con ventaja, especialmente en este país, donde coinciden al menos cinco "frenos" importantes para su desarrollo. El primero afecta al conjunto de la antropología y tiene que ver con el escaso conocimiento y reconocimiento de la disciplina en los diferentes ámbitos. En España, el asentamiento académico de la antropología social se ha producido hace relativamente poco tiempo, sobre todo si lo comparamos con otras ciencias sociales, y todavía hoy se desconocen una buena parte de sus posibilidades teóricas y prácticas. La comprensión limitada de su objeto estudio, asociado en el mejor de los casos al análisis de sociedades lejanas en tiempo e incluso espacio, ha contribuido no sólo a una caracterización errónea de la antropología, sino a disminuir las posibilidades de su proyección en el entramado social. Este desconocimiento se debe, en parte, al momento y el modo en que fue introducida, pero también al papel jugado por quiénes la practicamos desde entonces. La actitud proteccionista, y a menudo esencialista, de algunos antropólogos, no la ha beneficiado.

Definir el objeto de la antropología es relativamente sencillo si entendemos por ella la ciencia que describe, caracteriza e interpreta cualquier orden cultural en toda su complejidad estructural y funcional desde una perspectiva comparativa y holista. En la medida en que este objeto es vasto, y cada vez menos exclusivo de la antropología, estamos obligados a conocer qué se plantea desde otras instancias científicas, tal como se ha venido haciendo desde los orígenes de la disciplina, incluso a pesar de las fuertes tendencias que a lo largo del último siglo se han dado en torno a la segmentación y parcelación del conocimiento antropológico.

Uno segundo freno que debe evitarse es considerar que el interés antropológico por la alimentación es de segundo orden. Para muchos antropólogos, la comida y el comer no constituyen problemas relevantes para la teoría social y no son pocos los que relacionan el estudio de la necesidad humana de ingerir alimentos, y sus funciones y dimensiones biosociales, con la "pequeña" antropología. Nada más lejos de la realidad. Si, como ha sugerido Fernand Braudel (1979), la antropología se ha destacado por el interés que ha dedicado a los hechos sociales inscritos en la larga duración, tales como el lenguaje, el parentesco, la religión o el marco material, a los hechos que en definitiva competen a la evolución de la humanidad, la adaptación al entorno y la civilización, la alimentación tendría que haberse 
constituido en uno de ellos desde hace ya tiempo. Sin embargo, no ha sido así hasta hace relativamente poco o sólo ha sido así indirectamente.

Los conocimientos adquiridos por la antropología social sobre la alimentación en torno a otros objetos de estudio han sido numerosos, aunque es muy difícil poder articularlos antes de los años sesenta para hacer una antropología de la alimentación porque están extraordinariamente atomizados. Los trabajos antropológicos sobre alimentación han sido "de todos y de nadie" a la vez. Podemos encontrarlos repartidos en otros campos de estudio como la antropología económica, antropología ecológica, antropología del cuerpo, antropología política, antropología del desarrollo, antropología urbana, antropología de la religión, antropología del género, antropología de la medicina y la salud, etcétera. Pero la comida y el comer no ha sido para estos dominios más que un elemento de indexación de los factores sociales, de problemáticas más generales, como la identidad, el desarrollo, el ejercicio del poder, la división sexual de los roles sociales, la salud y la enfermedad, la marginación y la desigualdad. Hasta hace poco tiempo, pues, costaba hablar a priori de una antropología de la alimentación.

El tercer freno afecta especialmente a la antropología de la alimentación y tiene que ver con el hecho de que lo alimentario ha sido acaparado, en cuanto objeto de estudio científico, sobre todo por las ciencias biológicas y médicas. Esto ha sucedido, en general, en todo el mundo, aunque de forma más intensa en España. La consecuencia inmediata de esta situación ha sido la escasa demanda existente de antropólogos para el análisis las cuestiones socioculturales que afectan a los países industrializados. Simultáneamente, en tanto que ciencia social, comparte el resto de "males" con las otras disciplinas más allegadas: los científicos sociales no son invitados a participar en los diferentes proyectos de los que podrían realizar aportaciones debido a que su colaboración no se considera pertinente, o simplemente no se considera, y debido a que el propio científico social desconfía de sus potenciales y del uso que puede hacerse de su trabajo.

He señalado al inico de este texto que aunque son numerosas las disciplinas dedicadas al estudio de la alimentación humana dos son los enfoques predominantes, en primera instancia el biomédico y sociocultural, a los que habría que añadirle ciertamente otros dos más en tanto que últimamente han ido adquiriendo mayor peso, es el caso del cognitivo y tecnológico. Desde el primer enfoque se ha tendido a estudiar los determinismos fisiológicos y genéticos o las finalidades biológicas 
de la alimentación, así como las relaciones entre nutrición y salud, mientras que desde la segunda perspectiva se ha insistido en la dimensión simbólica, funcional e identificativa del hecho alimentario. Ha sido, no obstante, en esta última desde donde se ha planteado la oportunidad no sólo de tener en cuenta la multidisciplinaridad en el abordaje de lo alimentario sino también la colaboración interdisciplinar. El trabajo interdisciplinar implica un esfuerzo de comunicación, síntesis o integración, mientras que la multidisciplinaridad hace referencia mayormente a una recopilación, a un grupo o a un abanico de maneras de ver lo alimentario. A diferencia de otros científicos, los especialistas en antropología de la alimentación, como ya hicieran Audrey Richards y Margaret Mead en los años treinta y cuarenta, insisten repetidamente en la necesidad de romper los paradigmas de comunicación actuales entre los diferentes profesionales que se dedican a los temas de nutrición y alimentación, planteando como imperativo el hecho de contar siempre con la participación de las poblaciones donde se dirija cualquier acción de los expertos, con la finalidad de ejercer un verdadero intercambio y evitar crear cualquier dependencia en las culturas afectadas.

Hasta hace relativamente poco tiempo, el diálogo entre los dos tipos de ciencias, biomédicas y sociales, no ha sido frecuente, prácticamente inexistente, y si algo han mostrado las investigaciones que se han ido haciendo desde finales del siglo pasado es la patente dificultad por establecer un código común entre los dos ámbitos de observación y análisis. Los resultados de estos desencuentros entre lo biológico y lo social han sido, por un lado, los numerosos fracasos de los programas de intervención nutricional y salud y, por otro, las insuficiencias registradas en buena parte de los estudios sobre prácticas alimentarias que han insistido sólo en lo social, no pasando a veces del plano meramente descriptivo de las identidades culturales.

Cabe decir también, que a veces los enfoques unidisciplinares también se han producido en el propio seno de las ciencias humanas y sociales. Ciertamente, no es frecuente ver trabajar a los historiadores junto con los economistas o los sociólogos. Así, pues, una primera cuestión que hay que tener en cuenta es que las ciencias sociales no son unitarias (MURCOTT, 1998, p. 8-9). Existen en lo plural y consisten en la pluralidad de perspectivas intelectuales y de investigación, aunque compartan objetos, presupuestos y técnicas de análisis. Por otro lado, las ciencias sociales siempre serán diferentes del resto ciencias, simplemente en 
virtud de la naturaleza de aquello que estudian y que implica, de entrada, que nunca se pueda proceder por estricta replicación. No hay laboratorios, no hay experimentos. Los seres humanos tienen memoria y lenguaje, capacidades que aportan motivación y originalidad: ninguna circunstancia social es idéntica a otra, ninguna configuración política se puede reproducir nunca exactamente, ningún acontecimiento económico se repite del mismo modo. En este sentido, la historia no es justamente el pasado, sino también el presente y el futuro todo en uno, la historia es simplemente el transcurso del tiempo. La imposibilidad de replicación y de este modo la irrelevancia de una estricta versión del método experimental es una de las razones por la que una buena parte de los científicos sociales se distancian ellos mismos metodológicamente de las ciencias biomédicas, físicas u otras. Otros incluso se contrastan a sí mismos de los "científicos” y rehúsan denominar a su trabajo ciencia.

En realidad, el problema que tenemos muchos profesionales dedicados al estudio de la alimentación humana es que reconocemos la complejidad biosocial de la alimentación humana pero no la incorporamos en nuestros cuerpos teóricos y en nuestra praxis, o sólo muy parcialmente. En el fondo, el problema que tenemos la mayoría es que nos falta interés para plantearnos su análisis desde esta complejidad y, lo que es más importante, nos falta formación de base interdisciplinar que nos haga capaces de comprender y abordar el carácter multidimensional del hecho alimentario. Hay que insistir en que es necesario, dentro de las especializaciones, establecer un punto de encuentro, un espacio común donde los presupuestos teóricos y los recursos técnicos, en lugar de distanciarse, se aproximen y eviten, en la medida de lo posible, los errores y fracasos acumulados.

Un problema a resolver en este sentido, y que constituye otro freno importante, son las incómodas limitaciones que en el terreno aplicado imponen, con frecuencia, no sólo las diferentes disciplinas científicas entre sí, si no las propias áreas de conocimiento. A menudo los antropólogos más teóricos consideran que profesionalizarnos y trabajar para "resolver problemas" o "mejorar situaciones" puede acarrear excesivos costes epistemológicos y temen que la aplicabilidad convierta a la antropología en una ciencia excesivamente dependiente de las necesidades del "mercado científico".

Finalmente, otro freno reside en quienes nos dedicamos al estudio sociocultural de la alimentación y tiene que ver con nuestra capacidad, o interés, por articular 
este campo. Si bien vez disponemos de más herramientas de comunicación que nunca (listas de distribución, web temáticas, encuentros disciplinares), todavía es evidente el desconocimiento de los trabajos que, individualmente o colectivamente, se han llevado o se están llevando a cabo, la casi nula interconexión entre los proyectos de investigación vigentes y la ausencia de propuestas conjuntas. Parece como si, impelidos por las políticas universitarias pro-europeas, cada equipo o investigador tuviera únicamente interés por abrirse camino hacia el exterior, al margen o frente de los demás equipos españoles. Algunos parecen enfrascados en la carrera por la internacionalización de su docencia, investigación o disciplina y en el deseo de convertirse, en este contexto más amplio, en los especialistas y representantes locales.

No obstante, la convergencia con equipos europeos o internacionales no debería ser vista como una alternativa a la creación de mecanismos de articulación intraestatal de investigaciones o programas formativos, sino más bien como un proceso complementario. Los sucesivos congresos y jornadas de antropología y, en general de ciencias sociales, celebrados a lo largo de los últimos años han servido para que en el a veces encorsetado marco de simposios ya tradicionales, algunos de nosotros hayamos conocido el trabajo de los demás y para que se inicien, aunque tímidamente, los primeros proyectos de investigación conjunta. Aún así, se trata de iniciativas puntuales $y$, de momento, esporádicas ${ }^{14}$. Es evidente que habremos de dedicarnos más a la promoción de la colaboración interdisciplinar e interuniversitaria, en la medida en que el asentamiento de los estudios socioculturales sobre alimentación se producirá antes si en el ámbito académico e institucional español se unen esfuerzos que si éstos se producen de forma dispersa.

\section{Conclusión}

El futuro de la antropología de la alimentación en España está estrechamente relacionado con la capacidad de superar estos frenos. De todos ellos, y en el marco específico de los estudios sobre Alimentación, Salud y Cultura, quizá el más relevante sea el que tiene que ver con la interdisciplinaridad. Con frecuencia los esfuerzos interdisciplinares no han pasado de ser una declaración de buenas voluntades materializada, sobre todo, en las invitaciones que unos y otros científicos se hacen para participar en congresos, simposios, jornadas, estudios universitarios o tribunales de tesis. Entendemos que es difícil remontar ciertas 
tradiciones disciplinares, ciertas formas de ver las cosas, ciertas metodologías de investigación. Pero es hora ya de que la situación en la que estamos, básicamente estacionaria desde hace más de veinte años, sea superada por los intereses científicos particularistas y que, en lugar de estudiar la alimentación o la nutrición humana teniendo en cuenta exclusivamente la propia mirada, se incorporen, cuando así se requiera, las miradas que sobre el mismo tema están ofreciendo o pueden ofrecer, de forma paralela, otras disciplinas. Al fin y al cabo, nuestra sociedad está exigiendo a la comunidad científica la resolución de numerosos problemas alimentarios de origen multicausal que, consecuentemente, requieren respuestas interdisciplinares. Por lo demás, se trata de continuar los pasos que con anterioridad han seguido otros países y otras instituciones conscientes de la ineficacia generada por dichas limitaciones. Es obvio que los antropólogos que se decidan o, mejor dicho, que tengan la oportunidad de trabajar en el terreno de la alimentación y la salud pública deberán adquirir conocimientos especializados de carácter biomédico o psicológico en tanto que éstos, junto con su formación, son los que han de permitir abordar y dar solución, si cabe, a los diferentes problemas que hoy se plantean. De la misma forma, es preciso remontar la situación marginal que actualmente tiene la antropología o la sociología en la formación de los futuros especialistas en nutrición humana, sean facultativos o tecnólogos, ya que ellos van a ser con muchas probabilidades quienes acaben planificando, orientando y modificando los comportamientos alimentarios futuros.

\section{Referencias}

CACERES, J.; ESPEITX, E. Cuines en migració. Barcelona: Editorial Mediterrània, 2006. CARRASCO, S. Antropologia i alimentació. Una proposta per a l'estudi de la cultura alimentària. Bellaterra: S.P. Universitat Autònoma de Barcelona, 1992.

CASTRO, X. A lume manso: estudios sobre historia social da alimentación en Galicia. Vigo: Galaxia, 1998.

CONTRERAS, J.: Antropología de la alimentación, Madrid: Eudema, 1992.

CONTRERAS, J. (comp.): Alimentación y cultura. Barcelona: S.P. Universidad de Barcelona, 1995.

CONTRERAS, J.; GRACIA, M. (dirs.): La alimentación y sus circunstancias. Barcelona: Alimentaria Exhibitions, 2004.

CONTRERAS, J.; GRACIA, M. Alimentación y cultura. Perspectivas antropológicas. Barcelona: Ariel, 2005. 
CONTRERAS, J.; ESPEITX, E.; CACERES, J. Productes de la terra. Barcelona: Generalitat de Catalunya, 2001.

CONTRERAS, J.; RIERA, A.; MEDINA, X. Sabores del Mediterráneo. Barcelona: Institut de la Mediterrànea, 2005.

CONTRERAS, J.; GRACIA, M. (dirs.). Comemos como vivimos. Alimentación y estilos de vida saludables. Barcelona: Alimentaria Exhibitions, 2006.

CRUZ, J. Alimentación y cultura: Pamplona: Aunsa, 1991.

DIAZ, C. (coord.). ¿Cómo comemos? Cambios en los comportamentos alimentaros de los españoles. Madrid: Editorial Fundamentos, 2005.

DIAZ, C.; GOMÉZ, C. (coords.). Consumo, seguridad alimentaria y salud. Revista Internacional de Sociología, n. 40, 2005.

DIAZ, C.; GOMÉZ, C. (coords.). Alimentación, consumo y salud. Barcelona: Fundación "La Caixa”, 2008 (Colección Estudios Sociales, n. 24).

FIDALGO SANTAMARIA, X.A.; SIMAL GÁNDARA, J. (Eds.): Alimentación y cultura. Vigo:_Ayuntamiento de Celanova, 1993.

FISCHLER, C.; DE GARINE, I. Ciencias humanas y alimentación: tendencias actuales de la investigación europea. In: AINSWORTH, G. et al. Carencia alimentaria. Una perspectiva antropológica. Barcelona: Serbal/Unesco, 1988.

GARRIDO, A.; GARRIDO, A. (comp). Cultura alimentaria de España y América. Huesca: Val de Onsera, 1995.

GARRIDO, A. Comer cultura. Estudios de cultura alimentaria. Córdoba: Universidad de Córdoba, 2001.

GAONA, C.; NAVAS, J. Modelos alimentarios a debate: la interdisciplinaridad de la alimentación. Murcia: Universidad Católica San Antonio, 2003.

GONZALEZ TURMO, I. Comida de rico, comida de pobre. Sevilla: Universidad de Sevilla, 1995.

GONZALEZ TURMO, I.; ROMERO DE SOLIS, P. Antropología de la alimentación: nuevos ensayos sobre la dieta mediterránea. Sevilla: Universidad de Sevilla, 1996.

GONZALEZ TURMO, I. et al. Rojo y verde. Alimentación y cocinas en Marruecos.Gijón: Editorial Trea, 2007.

GRACIA, M. Paradojas de la alimentación contemporánea. Barcelona: Icaria, 1996(a).

GRACIA, M. Antropología de la alimentación. PRAT, J.; MARTÍNEZ, A. Ensayos de Antropología Cultural. Barcelona: Ariel, 1996(b).

GRACIA, M. La transformación de la cultura alimentaria. Cambios y permanencias en un contexto urbano. Madrid: Ministerio de Cultura,1997.

GRACIA, M. (coord.). Somos lo que comemos. Estudios de Alimentación y Cultura en España. Barcelona: Ariel, 2002 [2008]. 
GRACIA, M.; MILLÁN, A. (coords.). Alimentación, salud y cultura: enfoques antropológicos. Trabajo Socialy Salud, n. 51, 2005.

GRACIA, M.; COMELLES, J.M. (eds): No comerás. Narrativas sobre alimentación, cuerpo y género en el Nuevo Milenio. Barcelona: Icaria, 2007.

GRACIA, M. (dir.). Els trastorns alimentaris a Catalunya. Barcelona: Generalitat de Catalunya 2007 (Colección Estudis, n. 23).

GUIDONET, A. Antropología de la alimentación. Barcelona: Editorial UOC, 2007.

HUBERT, A. Anthropologie et recherce biomédicale. In: BARÉ, J.F. Les applications de l'anthropologie. París: Karthala, 1995.

ISOLETTA, S. (coord.): L'anorèxia com a simptoma social. Barcelona: Ediciones Experiencia, 2003.

KAPLAN, A.; CARRASCO, S. Migración, cultura y alimentación. Bellaterra: S.P. Universitat Autònoma de Barcelona, 1999.

KHARE, R.S. Food as nutrition and culture: notes towards an anthropological methodology. Social science information, v. 19, n. 3, p. 519-542, 1980.

MARTIN, E.; MORENO, J.L. Conflicto sobre lo sano. Un estudio de la alimentación en las clases populares en Andalucía. Sevilla: Consejería de Salud, 2005.

MEDINA, X (eds.). La alimentación mediterránea. Historia, cultura, nutrición. Barcelona: Iaria, 1996.

MEDINA, X. Alimentación, etnicidad y migración. Ser vasco y comer vasco en Cataluña. In: GRACIA, M. (coord). Somos lo que comemos. Barcelona: Ariel, 2002.

MEDINA, X. Food culture in Spain. Westport: Greenwood Publishing Group, 2005.

MENNELL, S et al. The sociology of food. Eating, diet and culture. Londres: Sage Publications, 1992.

MESSER, E. Anthropological perspectives on diet. Annual review of anthropology, v. 13, 1984.

MILLÁN, A. (comp.) Arbitrario cultural. Racionalidad e irracionalidad del comportamiento comensal. Huesca: Val de Onsera, 2004.

MURCOTT, A. The nation's diet. Londres. Longman, 1998.

MAUSS, M. Sociologie et anthropologie. París: PUF, 1950.

VVAA. El folk-lore Andaluz. Alimentación y Cultura en Andalucía, n. 9, 1992.

VVAA. Estudis d'historia agrària. Alimentació i Història, n. 13, 2000.

VVAA. Revista d'etnología de Catalunya. Dossier Alimentació i Cultura”, n. 2, 1993.

VVAA. Revista d'etnología de Catalunya. Dossier Transculturació, Consum i Alimentació, n. 17, 2000.

VVAA. Revista d'etnología de Catalunya. Dossier Biotecnología i Cultura, n. 27, 2005. 
VVAA. Abaco. Revista de cultura y ciencias sociales. Riesgos alimentarios y consumo sostenible, n. 31, 2002.

\section{Notas}

${ }^{1}$ Véanse los trabajos de Gracia (1996a), Gracia (2002) y Contreras y Gracia (2005).

${ }^{2}$ Utilizo el concepto de cultura alimentaria refiriéndonos al conjunto de actividades establecidas por los grupos humanos para obtener del entorno los alimentos que posibilitan su subsistencia, abarcando desde el aprovisionamiento, la producción, la distribución, el almacenamiento, la conservación y la preparación de los alimentos hasta su consumo, e incluyendo todos los aspectos simbólicos y materiales que acompañan las diferentes fases de este proceso.

${ }^{3}$ Para conocer la naturaleza de dichos esfuerzos pueden consultarse los trabajos de J. Goody, Cocinas, cocina y clases (Barcelona: Gedisa, 1995); A.Murcott, "Sociological and Social Anthropological Approaches to Food and Eating" (World Review of Nutrition and Diet. Basel: Karger, 1988); E. Messer, "Perspectivas antropológicas sobre la dieta" (in J. Contreras (comp). Alimentación y cultura. Barcelona: UB, 1995); G. Pelto, "Tendencias de la investigación en antropología nutricional” (VVAA. Carencia Alimentaria, Serbal/Unesco, Barcelona, 1988); C. Fischler y I. de Garine "Ciencias humanas y alimentación: tendencias actuales de la investigación europea” (in G. Ainsworth, et al.: Carencia alimentaria. Una perspectiva antropológica. Barcelona: Serbal/Unesco, 1988); E.L. Fürts et al. Palatable worlds: sociocultural food studies ( Oslo: Solum Forlag, 1991); S. Mennell; A. Murcott y A.H. Van Otterlo, The sociology of food Eating, diet and culture (Londres: Sage Publications, 1992); S. Carrasco, Antropologia i alimentació ( Publicacions d'Antropologia Social, UAB, Bellaterra, 1992); D. Maurer y J. Sobal, Eating agendas. Food an Nutrition as Social Problems ( Nueva Cork: Aldinde de Gruytier, 1995); D. Lupton, Food, the body and the self. Londres: Sage Publications, 1996; Wm. A. McIntosh, Sociologies and food and Nutrition (Nueva Cork: Plenum Publishing, 1996); A. Warde, Consumption, food and taste (Londres: Sage Publications, 1997); A. Beardsworth y T. Keil, Sociology on the menu (Londes: Routledge, 1997); P. Caplan (ed.), Food, health and identity (Londres: Routeledge, 1997); J. Germov y L. Williams, A Sociology of Food and Nutrition. The social appetite (Oxford: Oxford University Press, 1999); P. Atkins y I. Bowler, Food in society. Economy, culture, geography (Londres: Arnold, 2001); J.P. Poulain, Sociologies de l'alimentation (París: PUF, 2002); S.W. Mintz y Ch.Du Bois, "The Anthropology of food and eating” (Annual Review of Anthropology, v. 31, p. 99-119, 2002); H.Macbeth y J.MacClancy (eds.), Researching food habits. Methods and Problems (Oxford: Berghahn Books, 2004) y J. Contreras y M. Gracia, Alimentación y cultura. Perspectivas antropológicas (Barcelona: Ariel, 2005).

${ }^{4}$ Es el caso, por ejemplo, de los trabajos etnográficos de Gómez Pellón y Antonio Montesino en Cantabria o de Roberto González-Quevedo en Asturias. Para más referencias consúltese J. Prat (coord.): Investigadores e investigados: literatura antropológica en España desde 1954. Tarragona: Arxiu d'Etnografia de Catalunya, 1999.

${ }^{5}$ En relación con estos grupos, aquí sólo voy a mencionar las principales líneas de investigación que se han abierto hasta ahora, dejando para una futura ocasión la confección de un directorio que registre de forma exhaustiva las personas y los estudios hechos sobre Alimentación y Cultura. Es obvio que, además de estos equipos, hay antropólogos que comparten el mismo interés en otros lugares de la geografía española (Alicante, León, Galicia, País Vasco o Murcia) sin estar vinculados, necesariamente, a grupos más amplios. Otros trabajos de interés son los realizados en el País Vasco sobre la alimentación doméstica y que han sido recopilados por J.M. Barandiarán y A. Monterola en forma de atlas etnográfico, o los estudios llevados a cabo en los Museos de Antropología de las Islas Canarias sobre alimentación tradicional canaria y, en 
particular, sobre el gofio, y a sus investigadores principales, F. Estévez González y A. García Quesada.

Ejemplos de estos trabajos son los diferentes proyectos hechos en torno a los "Productos de la Tierra y Alimentos Tradicionales en la Isla de Tenerife", así como el "Inventario Etnográfico de los Molinos de Gofio" de la misma isla. Por su parte, en Galicia están los trabajos de X.A. Fidalgo de Santamariña sobre alimentación gallega y cultura (1993) o X. Castro, que ha publicado un libro sobre la historia social de la alimentación en Galicia (1998).

${ }^{6}$ La primera denominación de este equipo fue Grupo de Estudios Alimentarios. Más información sobre sus objetivos está disponible en http://www.odela-ub.com.

${ }^{7}$ Más información en http://web.mac.com/josepmcomelles/iWeb/REDAM/Pagina\%20principal. html.

${ }^{8}$ Más información en http://www.seiahs.info.

${ }_{9}^{9}$ Más información en http://moncayo.unizar.es/unizara/mapainvesaragon.nsf/ListaCompleta/131? OpenDocument.

${ }^{10}$ Más información sobre ICAF en http://erl.orn.mpg.de/-icaf/. En el Boletín de la sección europea de la Comisión Internacional de Antropología de la Alimentación (I.C.A.F.) correspondiente al volumen 1, n. 1, 1998, I. González Turmo daba cuenta de los diferentes equipos que estaban trabajando en España en aquellos momentos. Algunos de ellos han desaparecido y otros se han reestructurado sensiblemente.

${ }^{11}$ Más información disponible en http://sociologiadelaalimentacion.es.

${ }^{12}$ No pretendo ser exhaustiva a la hora de presentar la bibliografía sobre Alimentación y Cultura publicada en España. En los últimos años, han aparecido diversas recopilaciones bibliográficas que incluyen las principales publicaciones sobre antropología de la alimentación hasta 2000. Si bien éstas abordan el panorama a escala internacional, e incluso la primera de ellas incorpora obras sobre alimentación de carácter muy general, se pueden localizar sin dificultad las obras y artículos de los principales investigadores españoles: S. Carrasco, "Antropologia de l'alimentació: bibliografia", Revista d'Etnologia de Catalunya, n. 2, p. 78-85, 1992; J. A. Rubio Ardanaz, "Comer y leer, alimentos y publicaciones: producción bibliográfica en torno a la Antropología de la Alimentación” (Zainak, Cuadernos de Antropología-Etnografía, Donostia, n. 20, p. 257-278, 2000) y L. Cantarero, L.; M. Montejano; L.Abu-Shams, "Transculturació, alimentació i consum: una sel.lecció bibliogràfica" (Revista d'Etnologia de Catalunya, Barcelona, n. 17, noviembre, p. 140-149, 2000). Hay que apuntar, sin embargo, que son numerosas las publicaciones aparecidas entre el 2000-2008 y que, por tanto, otra tarea a realizar en un futuro será actualizar esta información.

${ }^{13}$ De Garine, I., "Une anthropologie alimentaire des français?” (Ethnologie française, París, v. 10, n. 3, 1980); S. Mennell et al., The sociology of food. Eating, diet and culture (Londres: Sage Publications, 1992).

${ }^{14}$ Una muestra de los diferentes tipos de trabajos producidos sobre Alimentación y Cultura desde las ciencias sociales ha quedado recogida en los dos volúmenes de actas del I Congreso Internacional de Alimentación y Cultura organizado por el Museo Nacional de Antropología en 1998 en Madrid (Huesca: Val de Onsera, 1999). También en la compilación realizada por Millán, A. (2004). Por su parte, en los próximos meses van a celebrarse el I Congreso Español de Sociología de la Alimentación (Gijón, mayo 2009) y el IX Coloquio Internacional REDAM. Alimentación, Salud y Cultura: Encuentros Disciplinares (Tarragona, junio 2009). 


\section{Alimentation and culture in Spain: an anthropology-based approach}

The food fact is essentially multidimensional: it goes between ecological, biological, psychological, socioeconomic or political spaces. As other complex phenomena, today no one can deny that food should be studied from a cross-disciplinary perspective. In Spain, there are many studies made from experimental, social or human sciences that, using hyper-specialized knowledge, show nutritional, psychological or economic dimensions, e.g., of food preferences and aversions, food consumption or malnutrition. However, they are usually unidirectional approaches of the same phenomenon, supported by the frameworks of each epistemic science. Scientific collaboration includes scarcely closer disciplines. But sparing scientific intersections does not seem, as I will try to show in this paper, appropriate or useful. Especially when it is necessary to understand and solve problems, that affect social groups, operate in multiple and changing contexts.

> Key words: food, culture, social anthropology, interdisciplinary, Spain. 